\title{
Perseverative thinking in depression and anxiety
}

\author{
Sonja Sorg ${ }^{1}$, Claus Vögele ${ }^{1}{ }^{*}$, Nadine Furka $^{2}$ and Andrea Hans Meyer ${ }^{3}$ \\ 1 Unité de Recherche INSIDE, University of Luxembourg, Walferdange, Luxembourg \\ 2 Department of Psychology, University of Leipzig, Leipzig, Germany \\ ${ }^{3}$ Division of Applied Statistics in Life Sciences, Department of Clinical Psychology and Psychotherapy, University of Basel, Basel, Switzerland
}

Edited by:

Colin G. DeYoung, University of

Minnesota, USA

Reviewed by:

Alexander Weiss, The University of

Edinburgh, UK

Markus Jokela, University of Helsinki,

Finland

\section{${ }^{*}$ Correspondence:}

Claus Vögele, Unité de Recherche INSIDE, Université du Luxembourg, Campus Walferdange, Route de

Diekirch - B.P. 2, L-7220 Walferdange,

Luxembourg.

e-mail: claus.voegele@uni.lu
The current study investigated the impact of worry and brooding as moderators of the tripartite model of depression and anxiety (TMDA). We hypothesized that both types of perseverative thinking would moderate the association between negative affectivity (NA) and both anxiety and depression. Complete data sets for this questionnaire survey were obtained from 537 students. Participants' ages ranged from 16 to 49 years with a mean age of 21.1 years $(S D=3.6)$. Overall, results from path analyses supported the assumptions of the TMDA, in that NA was a non-specific predictor for both depression and anxiety whilst lack of positive affectivity (PA) was related to depression only. Unexpectedly, perseverative thinking had an effect on the dependency of negative and positive affectivity. Worry was a significant moderator for the path NA-anxiety. All other hypothesized associations were only marginally significant. Alternative pathways as well as methodological implications regarding similarities and differences of the two types of perseverative thinking are discussed.

Keywords: depression, anxiety, perseverative thinking, rumination, brooding, worry

\section{INTRODUCTION}

About two-thirds of depressed individuals also meet the criteria for an anxiety disorder (Judd and Burrows, 1992). Clark and Watson's tripartite model of depression and anxiety (TMDA) is an affective model that aims to explain both the comorbidity and differentiation of depression and anxiety symptoms (Clark and Watson, 1991). In this model, anxiety and depression symptoms share a non-specific component that encompasses general affective distress (negative affectivity, NA). The syndromes can be distinguished by physiological hyperarousal $(\mathrm{PH})$ on the one hand, which is assumed to be specific for anxiety, and the relative absence of positive affectivity (PA) on the other hand, believed to be specific for depression. The model is both descriptive and prospective by explicating patterns of affectivity that predispose individuals to develop the respective disorders.

The TMDA has generally received good empirical support, with the strongest evidence coming from factor analytic studies across a variety of populations, for example child, undergraduate, community, and clinical samples (Watson et al., 1995; Joiner, 1996; Joiner et al., 1996; Lonigan et al., 2003; Tully et al., 2009). Nevertheless, there are also reports on inconsistencies and limitations of the model. For instance, the proposed specific role of low PA or anhedonia for the development of depressive symptoms is questionable as low PA is a factor in depression but also in some forms of anxiety, i.e., social phobia (Shankman and Klein, 2003).

Clark and Beck's cognitive content specificity hypothesis a product of Beck's cognitive theory - states that automatic thoughts or cognitive content should be related to emotional states (Beck, 1976; Clark et al., 1989). Following this view, anxious and depressive symptoms can be differentiated by unique cognitive

Abbreviations: NA, negative affectivity; PA, positive affectivity; $\mathrm{PH}$, physiological hyperarousal; TMDA, tripartite model of depression and anxiety. contents. In a meta-analytic review, Beck and Perkins (2001) evaluated the evidence for this hypothesis. They argued that - given the degree of shared variance between depression and anxiety constructs - the convergent symptomatology/cognition relationships should be stronger than the divergent ones. This was true for specific depressive contents across studies, while anxious cognitive content shared equal variance with depressive and anxious symptomatology. Also, in 5 out of the 13 studies included in the meta-analysis, anxious and depressive cognitive content was stronger interrelated than anxious and depressive symptoms, which weakens the hypothesis further. It can be argued that there are no cognitions that are truly specific to all anxiety disorders, emphasizing the problem of the heterogeneity of anxiety disorders.

Taken together, these results suggest that cognitive content alone might not be sufficient to explain depression and anxiety symptomatology. Rather, the way individuals engage in automatic cognitions may also be important for the two syndromes. For example, perseverative thinking in the form of depressive rumination and anxious worry has been shown to be related to depression and anxiety symptomatology, respectively. Depressive rumination has been defined as passively and repetitively focusing on symptoms of distress and the circumstances surrounding those symptoms, and can be understood as a stable emotional response style (Just and Alloy, 1997; Bagby et al., 2004). Rumination has been shown to intensify depressed mood, predict the onset, recurrence, severity, and duration of depressive episodes as well as suicidal ideation. It interferes with problem solving, leads to more pessimistic future perspectives and less social support (Morrow and Nolen-Hoeksema, 1990; Nolen-Hoeksema and Morrow, 1991; Lyubomirsky and Nolen-Hoeksema, 1995; Nolen-Hoeksema and Davis, 1999; Bagby et al., 2004; Miranda and Nolen-Hoeksema, 2007). 
There is growing evidence that at least two forms of rumination with distinct effects on depression can be distinguished: brooding and reflection. Brooding or passively dwelling on symptoms of distress has been found to be the key factor in the prediction of depressive symptoms. Self-reflection or actively attempting to gain insight into problems, on the other hand, is not related to any negative outcomes (Treynor et al., 2003; Burwell and Shirk, 2007; Moberly and Watkins, 2008). Therefore, studies on the detrimental effects of rumination should rely preferably on the brooding dimension of the construct.

Rumination and its maladaptive subtype brooding share common elements with worry, another form of perseverative thinking. Worry can be defined as "repetitive, uncontrollable thoughts about potential negative life events" (Segerstrom et al., 2000). It is a cardinal feature of general anxiety disorder (GAD) which is often co-morbid with depression (American Psychiatric Association, 2000). The perseverative nature of both rumination and worry entails a similarity between these two constructs, whilst the content of them appears to differ with rumination being past oriented and worry being future oriented (Papageorgiou and Wells, 2004). Nevertheless, there is no consensus on the specificity of the two constructs in the literature. Most studies investigating perseverative thinking link rumination specifically to depression, and worry to anxiety. Far fewer studies have investigated the contribution of both forms of perseverative thinking to both syndromes (NolenHoeksema, 2000; Fresco et al., 2002; Garnefski et al., 2002; Sarin et al., 2005). Segerstrom et al. (2000), for example, found that repetitive thought was related to both anxiety and depression; specific forms of repetitive thinking such as worry and rumination, however, did not predict anxiety or depression by themselves. Given these findings, perseverative thinking might be especially relevant for mixed depression/anxiety symptoms. To our knowledge, the role of perseverative thinking within the TMDA has not been investigated yet.

For the present study we used a sample of adults that were slightly younger than the average age at onset of Major Depressive Disorder (American Psychiatric Association, 2000). The aim in doing so was to investigate the prospective properties of the TMDA for individuals that are at an at-risk age for the development of this disorder.

We hypothesize that both depressive brooding and anxious worry moderate the associations as predicted by the TMDA, by strengthening the associations between NA, and anxiety and depression (Figure 1). We hypothesize further that there will be no specific contribution of either brooding or worry as moderators of the TMDA, as we assume that the moderating properties of brooding and worry are due to the higher-order factor perseverative thinking.

\section{MATERIALS AND METHODS PARTICIPANTS AND DESIGN}

For this questionnaire survey, 537 (477 female) undergraduate students were recruited from the student population of a British University. Complete data sets were obtained from 508 students. Participants' age ranged from 16 to 49 years with a mean age of 21.1 years $(\mathrm{SD}=3.6)$. Most of the student volunteers indicated being British (64.7\%), followed by Indian (7.8\%), other European (4.7\%), Caribbean (3.5\%), Irish (2.1\%), and other (3.9\%). Seventy

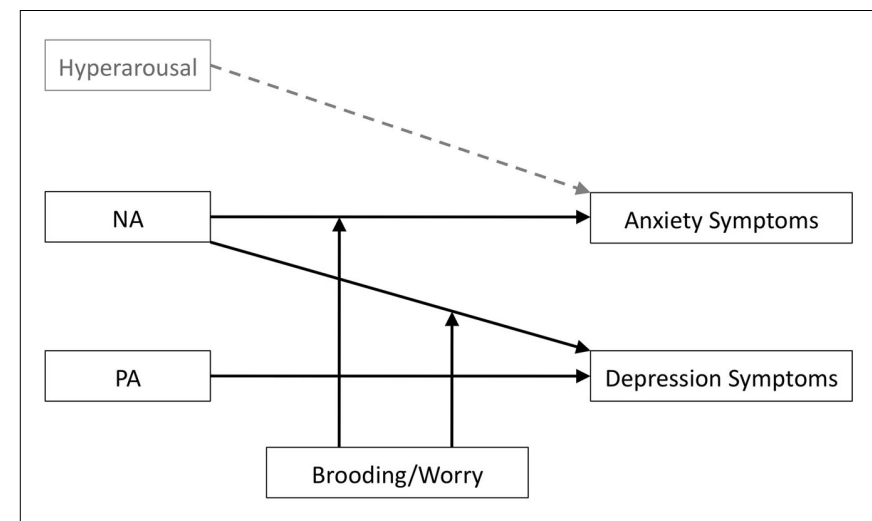

FIGURE 1 | Hypothesized model. Brooding and Worry moderate the paths between negative affectivity and both depression and anxiety.

students (13.6\%) did not indicate their ethnic background. Students were recruited from non-psychology courses in order to avoid confounding effects due to familiarity with the topics investigated. Ethical approval was obtained from the University's ethics board.

\section{MEASURES}

The Positive and Negative Affect Scale (PANAS, trait version; Watson et al., 1988) provides a brief, reliable, valid, and stable measure of trait affectivity. This questionnaire is the standard instrument for measuring trait affect as proposed in the TMDA and has been developed with and applied on undergraduate student samples. It consists of 20 items, half of which measure positive and negative affect, respectively.

The disposition to engage in brooding responses to sad mood was assessed using a revised version of the Ruminative Response Scale (RRS) of the Response Styles Questionnaire (RSQ). For this scale, items of the original RRS that were contaminated with depressive symptoms were omitted. The revised RRS consists of 10 items and loads on two factors: brooding and reflection. Participants indicated how they generally react when in a depressed mood with a range from 0 (never) to 3 (always). The revised version is a reliable and valid measure of both the brooding and reflective subtype of depressive rumination, as was shown in investigations with healthy and clinical populations of all age groups including student samples (Kasch et al., 2001; Treynor et al., 2003). For this study, only the brooding dimension was used for analysis.

Anxious worry was assessed using the Penn State Worry Questionnaire (PSWQ, trait version; Meyer et al., 1990) which has been extensively applied and validated. The PSWQ has shown good reliability in student samples (Fresco et al., 2003).

\section{Depressive symptoms}

The Center for Epidemiologic Studies Depression Scale (CES-D; Radloff, 1977) is a standardized and extensively used instrument to measure depression in the general population. It is the most commonly used instrument in depression research with young adult samples. The scale consists of 20 items and has been extensively validated. The psychometric properties have been shown to be satisfactory. 
Table 1 | Means, SD, range, and reliability for all measures.

\begin{tabular}{|c|c|c|c|c|c|c|}
\hline & PA & NA & Brooding & PSWO & BAI & CES-D \\
\hline$n$ & 523 & 523 & 532 & 518 & 497 & 510 \\
\hline Mean/SD & $3.50(0.57)$ & $2.09(0.64)$ & $1.30(0.66)$ & $3.21(0.88)$ & $1.56(0.50)$ & $0.83(0.55)$ \\
\hline Range & $13-49$ & $17-80$ & $0-15$ & $17-80$ & $21-74$ & $0-55$ \\
\hline Cronbach's $\alpha$ & 0.830 & 0.835 & 0.782 & 0.929 & 0.923 & 0.908 \\
\hline
\end{tabular}

Table 2 | Zero-order correlations between variables.

\begin{tabular}{|c|c|c|c|c|c|}
\hline & NA & Brooding & PSWO & BAI & CES-D \\
\hline NA & & $0.491 * *$ & $0.426^{* *}$ & $0.471 * *$ & $0.474 * *$ \\
\hline PSWO & & & & $0.485^{* *}$ & $0.532 * *$ \\
\hline BAI & & & & & $0.661 * *$ \\
\hline
\end{tabular}

${ }^{* *} p<0.001$.

\section{Anxiety symptoms}

Beck's anxiety inventory (BAI; Beck et al., 1988) was originally developed for the assessment of clinical anxiety, but has been shown to be the superior instrument for anxiety assessment in non-clinical undergraduate samples as well (Creamer et al., 1995). The 21-item scale has high internal consistency and high discriminant validity to depression in student samples.

\section{STATISTICAL ANALYSIS}

For all scales, total sums were computed and used for analysis. To test the proposed causal relationships (Figure 1) between PA/NA and the TMDA dimensions, path analyses were conducted (Kline, 2005). To assess moderating effects of brooding and worry we used multigroup analyses. Multigroup analyses allow for the assessment of the question whether specific path coefficients, a group of path coefficients, or all path coefficients of a path model simultaneously differ between groups. We divided each of the two scales for brooding and worry into two groups based on a median split. The path model was thus analyzed for high/low brooding ruminators and high/low worriers in order to detect moderating effects of perseverative thinking for specific path coefficients and for all path coefficients simultaneously. PA and NA were exogenous variables in this model, anxiety and depression symptoms were endogenous variables.

Preliminary analyses were conducted excluding data from male participants (11\%) and compared with results from the whole sample. There were no differences in anxiety or depression scores between men and women, nor did the results of the path analyses differ when excluding men from those including men and women. Results are, therefore, reported for the entire sample. Scales were transformed (log, square root) if necessary to meet regression analysis assumptions.

All analyses were conducted using the software package AMOS 18.

\section{RESULTS}

Means, SDs, ranges, and scale reliabilities of all measures are shown in Table 1. As the path model was just identified, no fit statistics are shown. Zero-order correlations between all variables are shown in Table 2. All variables were significantly interrelated, with $p$-values below 0.01 for all measures $(n=506)$. Perseverative thinking was more closely related to NA than to PA (e.g., brooding-NA: $r=0.491$; brooding-PA: $r=-0.148)$. Depression and anxiety symptoms were highly related $(r=0.661)$. Both kinds of perseverative thinking had strong associations with depression as well as with anxiety (e.g., PSWQ-CES-D: $r=0.532$ ).

\section{PATH ANALYSES}

\section{Overall model}

All four coefficients significantly differed from zero although the coefficient PA-BAI was the least significant $(\beta=-0.093$, $p=0.019)$. The other three coefficients had absolute $\beta$-values between 0.28 and 0.45 and $p$-values of $<0.001$ each. The correlation between PA and NA was $r=-0.18(p<0.001)$. Anxiety and depression symptoms were strongly related $(r=0.57, p<0.001)$.

\section{Subgroups worry low/high}

Taken together, the four path coefficients significantly differed between the two subgroups $(p=0.016)$. This was primarily due to the coefficient NA-anxiety symptoms, which was strongly and significantly increased in the "high worry" subgroup compared to the "low worry" subgroup ( $p<0.001$, constraining the three other coefficients to be equal between subgroups), whereas the other three coefficients did not differ between subgroups $(p>0.40$ for each coefficient). Note that when using three instead of two subgroups (low, medium, and high worry), results for the coefficient NA-anxiety symptoms were similar with the highest coefficients for the "high worry" and the lowest coefficients for the "low worry" subgroup, suggesting a linear association. The correlations between NA and PA were $r=-0.02(p=0.75)$ and $r=-0.25$ 


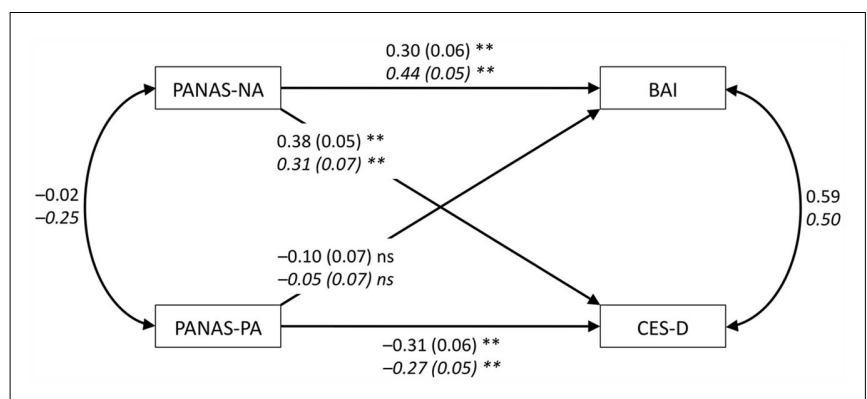

FIGURE 2 | Path- and correlation coefficients in low (high) worry-groups. Numbers of the high worry group are in italics in the lower lines. The numbers in parentheses are SEs of path coefficients.

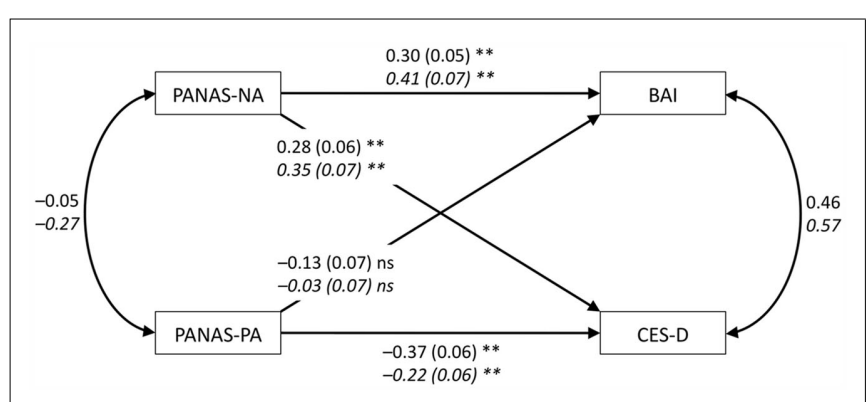

FIGURE 3 | Path- and correlation coefficients in low (high) brooding groups. Numbers of the high brooding group are in italics in the lower lines. The numbers in parentheses are SEs of path coefficients.

$(p=0.001)$ for low and high worry, respectively. For the subgroup "low worry," all but one coefficient significantly differed from 0 (absolute $\beta$ between 0.30 and $0.38, p<0.001$ ) with the exception of the path PA-BAI $(\beta=-0.10, p=0.084)$. The correlation between BAI and CES-D was $0.59(p<0.001)$. For the subgroup "high worry," all but one coefficient significantly differed from 0 (absolute $\beta$ between 0.27 and $0.44, p<0.001$ ), the exception was PA-BAI $(\beta=-0.05, p=0.44)$. The correlation between BAI and CES-D was $0.50(p<0.001$; Figure 2$)$.

\section{Subgroups RSQ brooding low/high}

For both groups, all path coefficients differed significantly from zero with the exception of PA-BAI in the high brooding group $(\beta=-0.03, p=0.65)$. Considered together, the four path coefficients did not differ significantly between the two subgroups $(p=0.084)$. In addition, none of the four coefficients differed between the two subgroups, although the path coefficient NAanxiety was 0.41 for the high versus 0.30 for the low brooding group ( $p>0.05$ for each coefficient, constraining the three other coefficients to be equal between subgroups). The relationship between PA and NA was stronger in the high brooding group (brooding low: $r=-0.05, p=0.36$; brooding high: $r=-0.27$, $p<0.001$; Figure 3).

\section{DISCUSSION}

The main aim of the present study was to investigate the impact of brooding and worry as moderators of the association between NA and depression and anxiety in a sample of young adults. The results support the assumptions of the TMDA, in that NA predicted both anxiety and depressive symptoms. Low PA also significantly predicted both anxiety and depression, but the association between low PA and anxiety was negligible, thus confirming the proposed specific role of low PA for depression.

Results regarding the moderating role of brooding and worry were mixed. Although both types of perseverative thinking moderated the associations between NA and both anxiety and depression in the hypothesized direction, this was statistically significant for worry only. As hypothesized, worry moderated the predicted association by strengthening the path NA-anxiety. The hypothesis that both kinds of perseverative thinking moderate both paths NAdepression/NA-anxiety symptoms, therefore, received only partial support. Nevertheless, even though brooding was not a significant path moderator, it still had a strong tendency to strengthen the association between NA and anxiety. Neither worry nor brooding had a significant impact on the path NA-depression. While worry was a significant specific path moderator, brooding moderated both paths NA-worry and NA-depression only marginally. In the present study, worry had a specific and strong effect, while the moderating effect of brooding was non-specific and weak.

Segerstrom et al. (2000) found a stronger non-specific effect in non-depressed students, while specific associations between rumination and depression were found in patients only. It could be argued that the general proneness to perseverative thinking is a predisposing factor for both anxiety and depression, which may develop into the respective clinical syndromes when filled with specific content. This notion is also supported by the high intercorrelations between brooding, worry, anxiety, and depression in our non-clinical sample. In the future, it should be investigated whether interventions to reduce perseverative thinking would be effective for the prevention of both disorders. However, in individuals with clinical depression or anxiety, it could be useful to target rumination and worry specifically. Even though the impact of perseverative thinking in the present path model was mixed, zero-order correlations between worry, brooding, depression, and anxiety were high. This suggests that there may be other pathways that connect perseverative thinking with depression and anxiety. There is preliminary evidence that higher-order cognitions like beliefs about rumination and worry may explain associations between perseverative thinking and psychopathology. In their review, Papageorgiou and Wells (2004) argue that in addition to content and process, negative beliefs about worry and rumination link perseverative thinking with psychopathology. This approach should be followed up in future research.

In individuals reporting high levels of perseverative thinking there was a significant negative association between NA and PA. Unexpectedly, in participants with low levels of perseverative thinking no such association was found. To our knowledge, there is no literature on the impact of brooding and worry on the association of NA and PA. Diener and Emmons (1984) found the association between NA and PA to depend on the time frame assessed: when measuring momentary affect, PA and NA were inversely related. This association diminished linearly when longer intervals were evaluated. In another study (Johnson et al., 2008), only students with bipolar disorder ruminated in response to 
positive affect compared to students with unipolar depression or students with no disorder. It is possible, therefore, that the tendency to ruminate or worry predominantly leads most individuals to exacerbate their negative affect only. Following Diener's findings (1984) that it does not seem possible to experience positive and negative affect at the same time, one could apply this result to people who experience negative affect for longer periods. People with high levels of rumination have prolonged periods of negative affect (Nolen-Hoeksema, 1991; Lyubomirsky and Tkach, 2004). During these prolonged periods, it is unlikely for these individuals to experience positive affective states. This way, the negative association between NA and PA in participants with high levels of perseverative thinking might occur. Another explanation could be that worriers' and ruminators' access to the recall of their general affect could be biased and influence the PANAS scores toward the more negative (Lyubomirsky and Nolen-Hoeksema, 1995; Lyubomirsky et al., 1998). In order to obtain more valid data, these processes should be investigated with ecologically momentary assessment methods.

\section{LIMITATIONS}

There could be methodological reasons for the rather weak moderating properties of brooding on the associations between NA and anxiety/depression. One concern lies in the definition of rumination and, therefore, the wording of the brooding items of the RSQ. Thoughts assessed by the RSQ focus on the content of the respective cognition but not on their perseverative nature. Although the internal consistency and predictive validity of the RSQ for depression have been demonstrated, there are no data on the construct validity of the brooding subscale. In the present study, the subscale had the lowest internal consistency of all measures employed (see Table 1). Similar problems were reported by Johnson et al. (2008) who found no association between the brooding subscale of the RSQ and depression scores in participants without mood disorders. For future research, instruments that reliably assess the perseverative aspect of depressive rumination are needed. Similarly, when comparing definitions of rumination and worry, there are differences regarding the controllability of the two. The dimension of uncontrollability is a defining criterion for worry but not brooding, and it might be beneficial for future research to emphasize the aspect of uncontrollability for brooding as well.

\section{REFERENCES}

American Psychiatric Association. (2000). Diagnostic and Statistical Manual of Mental Disorders. Fourth Edn, Washington, DC: American Psychiatric Association.

Bagby, R. M., Rector, N. A., Bacchiochi, J. R., and McBride, C. (2004). The stability of the response styles questionnaire rumination scale in a sample of patients with major depression. Cognit. Ther. Res. 28, 527-538.

Beck, A. T. (1976). Cognitive Theory and the Emotional Disorders. New York: International Universities Press.

Beck, A. T., Epstein, N., Brown, G., and Steer, R. A. (1988). An inventory for measuring clinical anxiety:

Papageorgiou and Wells (1999), for example, report that in patients with major depressive disorder and panic disorder, rumination was rated longer in duration, and less controllable than worrying. Watkins et al. (2005) found that rumination and worry share the same processes although different content. Sharpening the definition of rumination in a way that the aspects of uncontrollability or perseveration are emphasized would contribute to improving instruments assessing brooding.

Another limitation regarding the external validity of the present results lies in the sample investigated. To capture factors underlying depression and anxiety we focused mostly on young adults that were younger than the average ages of onset for anxiety (25 years) and depression (30-40 years, American Psychiatric Association, 2000). The results are relevant from the clinical perspective of a higher prevalence of these disorders in young adults but may not apply to older individuals or clinical samples. There is some evidence that the mechanisms linking perseverative thinking and mood or anxiety disorders differ between student populations and clinical samples. In clinical samples, the specific associations between rumination and depression seem much stronger (Segerstrom et al., 2000; Johnson et al., 2008). If this was confirmed in future research, specific implications for prevention and intervention could be drawn. For the prevention of anxiety and depression, the reduction of general perseverative thinking might be a useful target. For the intervention of clinical depression and anxiety, brooding and worry should be targeted specifically. Another potential limitation was the skewed gender ratio. To address this issue, we conducted all analyses for the female sample only, with no impact on the results.

\section{CONCLUSION}

The double role of perseverative thinking, i.e., specific and general, should attract more attention in future investigations. The present result that brooding had a stronger moderating impact on the path NA-anxiety than on the path NA-depression emphasizes this point. The current findings suggest that the assumption of the cognitive content specificity hypothesis should be extended. It is not only the contents of automatic cognitions that have detrimental effects on mental health but also the perseverative nature of these cognitions. More research in this area could have implications for the prevention and treatment of anxiety and depression.

Clark, L. A., and Watson, D. (1991). Tripartite model of anxiety and depression: psychometric evidence and taxonomic implications. $J$. Abnorm. Psychol. 100, 316-336.

Creamer, M., Foran, J., and Bell, R. (1995). The beck anxiety inventory in a non-clinical sample. Behav. Res. Ther. 33, 477-485.

Diener, E., and Emmons, R. A. (1984). The independence of positive and negative affect. J. Pers. Soc. Psychol. 47, 1105-1117.

Fresco, D. M., Frankel, A. N., Mennin, D. S., Turk, C. L., and Heimberg, R. G. (2002). Distinct and overlapping features of rumination and worry: the relationship of cognitive production to negative affective states. Cognit. Ther. Res. 26, 179-188.

Fresco, D. M., Mennin, D. S., Heimberg, R. G., and Turk, C. L. (2003). Using the Penn State Worry Questionnaire to identify individuals with generalized anxiety disorder: a receiver operating characteristic analysis. $J$. Behav. Ther. Exp. Psychiatry. 34, 283-291.

Garnefski, N., Legerstee, J., Kraaij, V., van den Kommer, T., and Teerds, J. (2002). Cognitive coping strategies and symptoms of depression and anxiety: a comparison between adolescents and adults. J. Adolesc. 25, 603-611. 
Johnson, S. L., McKenzie, G., and McMurrich, S. (2008). Ruminative responses to negative and positive affect among students diagnosed with bipolar disorder and major depressive disorder. Cognit. Ther. Res. 32, 702-713.

Joiner, T. E. (1996). A confirmatory factor-analytic investigation of the tripartite model of depression and anxiety in college students. Cognit. Ther. Res. 20, 521-539.

Joiner, T. E., Catanzaro, S. J., and Laurent, J. (1996). Tripartite structure of positive and negative affect, depression, and anxiety in child and adolescent psychiatric inpatients. $J$. Abnorm. Psychol. 105, 401-409.

Judd, F. K., and Burrows, G. D. (1992). "Anxiety disorders and their relationship to depression," in Handbook of Affective Disorders, 2nd Edn, ed. E. S. Paykel (New York: Guilford Press), 77-87.

Just, N., and Alloy, L. B. (1997). The response styles theory of depression: tests, and an extension of the theory. J. Abnorm. Psychol. 106, 221-229.

Kasch, K. L., Klein, D. N., and Lara, M. E. (2001). A construct validation study of the response styles questionnaire rumination scale in participants with a recent-onset major depressive episode. Psychol. Assess. 13, 375-383.

Kline, R. B. (2005). Principles and Practice of Structural Equation Modeling, 2nd Edn. New York: Guilford Press.

Lonigan, C. J., Phillips, B. M., and Hooe, E. S. (2003). Relations of positive and negative affectivity to anxiety and depression in children: evidence from a latent variable longitudinal study. J. Consult. Clin. Psychol. 71, 465-480.

Lyubomirsky, S., Caldwell, N. D., and Nolen-Hoeksema, S. (1998). Effects of ruminative and distracting responses to depressed mood on retrieval of autobiographical memories. J. Pers. Soc. Psychol. 75, 166-177.

Lyubomirsky, S., and Nolen-Hoeksema, S. (1995). Effects of self-focused rumination on negative thinking and interpersonal problem solving. J. Pers. Soc. Psychol. 69, 176.

Lyubomirsky, S., and Tkach, C. (2004). "The consequences of dysphoric rumination," in Depressive Rumination - Nature, Theory and Treatment, eds C. Papageorgiou and A. Wells (Chichester: John Wiley \& Sons Ltd.), 21-41.

Meyer, T. J., Miller, M. L., Metzger, R. L., and Borkovec, T. D. (1990). Development and validation of the Penn State Worry Questionnaire. Behav. Res. Ther. 28, 487-495.

Miranda, R., and Nolen-Hoeksema, S. (2007). Brooding and reflection: rumination predicts suicidal ideation at 1-year follow-up in a community sample. Behav. Res. Ther. 45, 3088-3095.

Moberly, N. J., and Watkins, E. R. (2008). Ruminative self-focus and negative affect: an experience sampling study. J. Abnorm. Psychol. 117, 314-323.

Morrow, J., and Nolen-Hoeksema, S. (1990). Effects of responses to depression on the remediation of depressive affect. J. Pers. Soc. Psychol. 58, 519-527.

Nolen-Hoeksema, S. (1991). Responses to depression and their effects on the duration of depressive episodes. J. Abnorm. Psychol. 100, 569-582.

Nolen-Hoeksema, S. (2000). The role of rumination in depressive disorders and mixed anxiety/depressive symptoms. J. Abnorm. Psychol. 109, 504-511.

Nolen-Hoeksema, S., and Davis, C. G. (1999). "Thanks for sharing that": ruminators and their social support networks. J. Pers. Soc. Psychol. 77, 801-814.

Nolen-Hoeksema, S., and Morrow, J. (1991). A prospective study of depression and posttraumatic stress symptoms after a natural disaster: the 1989 Loma Prieta earthquake. J. Pers. Soc. Psychol. 61, 115-121.

Papageorgiou, C., and Wells, A. (1999). "Dimensions of depressive rumination and anxious worry: a comparative study," in Thirty-Third Annual Convention of the Association for Advancement of Behavior Therapy, Toronto.

Papageorgiou, C., and Wells, A. (2004). "Nature, functions, and beliefs about depressive rumination," in Depressive Rumination: Nature, Theory and Treatment, 1st Edn, eds C. Papageorgiou and A. Wells (Chichester: John Wiley \& Sons Ltd.), 3-20.

Radloff, L. S. (1977). The CES-D scale: a self-report depression scale for research in the general population. Appl. Psychol. Meas. 1, 385-401.

Sarin, S., Abela, J., and Auerbach, R. (2005). The response styles theory of depression: a test of specificity and causal mediation. Cogn. Emot. 19, 751-761.

Segerstrom, S. C., Tsao, J. C. I., Alden, L. E., and Craske, M. G. (2000). Worry and rumination: repetitive thought as a concomitant and predictor of negative mood. Cognit. Ther. Res. 24, 671-688.

Shankman, S. A., and Klein, D. N. (2003). The relation between depression and anxiety: an evaluation of the tripartite, approach-withdrawal and valence-arousal models. Clin. Psychol. Rev. 23, 605-637.

Treynor, W., Gonzalez, R., and NolenHoeksema, S. (2003). Rumination reconsidered: a psychometric analysis. Cognit. Ther. Res. 27, 247-259.

Tully, P. J., Zajac, I. T., and Venning, A. J. (2009). The structure of anxiety and depression in a normative sample of younger and older Australian adolescents. J. Abnorm. Child. Psychol. 37, 717-726.

Watkins, E., Moulds, M., and Mackintosh, B. (2005). Comparisons between rumination and worry in a non-clinical population. Behav. Res. Ther. 43, 1577-1585.

Watson, D., Clark, L. A., and Tellegen, A. (1988). Development and validation of brief measures of positive and negative affect: the PANAS scales. $J$. Pers. Soc. Psychol. 54, 1063-1070.

Watson, D., Clark, L. A., Weber, K., Smith Assenheimer, J., Strauss, M. E. and McCormick, R. A. (1995). Testing a tripartite model: II. Exploring the symptom structure of anxiety and depression in student, adult, and patient samples. J. Abnorm. Psychol. 104, 15.

Conflict of Interest Statement: The authors declare that the research was conducted in the absence of any commercial or financial relationships that could be construed as a potential conflict of interest.

Received: 09 September 2011; accepted: 15 January 2012; published online: 13 February 2012.

Citation: Sorg S, Vögele C, Furka $N$ and Meyer AH (2012) Perseverative thinking in depression and anxiety. Front. Psychology 3:20. doi: 10.3389/fpsyg.2012.00020

This article was submitted to Frontiers in Personality Science and Individual Differences, a specialty of Frontiers in Psychology.

Copyright (c) 2012 Sorg, Vögele, Furka and Meyer. This is an open-access article distributed under the terms of the Creative Commons Attribution Non Commercial License, which permits noncommercial use, distribution, and reproduction in other forums, provided the original authors and source are credited. 